\title{
The effect of local corticosteroid injection on F-wave conduction velocity and sympathetic skin response in carpal tunnel syndrome
}

\author{
Orhan Deniz $\cdot$ Recep Aygül $\cdot$ Dilcan Kotan • \\ Gökhan Özdemir · Faruk Ömer Odabaş • \\ M. Dursun Kaya $\cdot$ Hızır Ulvi
}

Received: 4 September 2010 / Accepted: 30 December 2010 / Published online: 29 January 2011

(C) The Author(s) 2011. This article is published with open access at Springerlink.com

\begin{abstract}
The aim of this study was to evaluate the efficacy of steroid injection for the treatment of the carpal tunnel syndrome (CTS), with F-wave parameters and sympathetic skin response (SSR). Seventeen hands of 10 women patients were treated with local steroid injection with 2-month follow-up. All patients underwent single injection into the carpal tunnel. Response to injection was measured nerve conduction studies (NCSs), median nerve $\mathrm{F}$ waves, and SSR before and after treatment. To determine the normal values, 42 hands of 21 healthy women were also studied. There was a significant improvement of sensory and motor nerve conduction values when compared to baseline values $(P<0.01)$. At the end of follow-up period, the median sensory distal latency and the sensory latency differences between the median and the ulnar nerve were improved 35 and $65 \%$, respectively. The maximum, mean F-wave amplitudes and chronodispersion showed a slight improvement with respect to baseline values and controls,
\end{abstract}

O. Deniz

Department of Neurology,

Ataturk Education and Research Hospital, Ankara, Turkey

R. Aygül · D. Kotan $(\bowtie) \cdot$ G. Özdemir · H. Ulvi

Department of Neurology,

Ataturk University Faculty of Medicine,

25240 Erzurum, Turkey

e-mail: dilcankotan@yahoo.com

F. Ö. Odabaş

Department of Neurology,

Marasal Cakmak Military Hospital, Erzurum, Turkey

M. D. Kaya

Division of Computer Programming,

Atatürk University Vocational High School,

Erzurum, Turkey but statistical significance was not achieved after treatment. Although no statistically significant improvements were observed in SSR parameters, slightly decreased amplitudes and increased habituation of SSR were noted at the end of the treatment. The present study shows that the local steroid injection results in improvement in NCSs values, but the Fwave parameters were not effectual in short-term outcome of CTS treatment. These findings suggest that the sensory latency differences between the median and the ulnar wristto-digit 4 are better parameters in the median nerve recovery after treatment than the median sensory distal latency. Furthermore, the SSR does not seem to be a sensitive method in follow-up of CTS treatment.

Keywords Carpal tunnel syndrome - Steroid injection . Median nerve F waves - Nerve conduction studies .

Sympathetic skin response

\section{Introduction}

The carpal tunnel syndrome (CTS), caused by compression of the median nerve at the wrist, is considered the most common entrapment neuropathy in adults, particularly in women. It is an important mononeuropathy that causes neuropathic pain and neurologic symptoms in the hand [1,2]. Non-surgical treatment for CTS is frequently offered to those with mild to moderate symptoms. Steroid injection into the wrist is often successful. It may cause complete or significant pain relief in $60-70 \%$ of patients for a longer period $[3,4]$.

Several tools have been used to assess the outcome of CTS treatment. Median motor distal latency (mMDL) and sensory nerve conduction velocity (mSNCV) were being widely accepted as the most sensitive for measuring 
changes in CTS [5-7]. Comparative tests of sensory nerve latency between the median nerve and the ulnar nerve are well documented in the literature [8]. Chang et al. [9] found that in patients with normal sensory and motor conduction velocities, measuring the latency between the median and the ulnar nerve for the ring finger increases the diagnostic yield by $10 \%$. Therefore, this change may be more sensitive for assessing the outcome of treatment.

Since the description of F-wave has found a wide application in the assessment of peripheral nerve lesions, $\mathrm{F}$ waves have been used to evaluate proximal motor nerve conduction and excitability of the motor neuron pool. Their analysis is very useful in clinical neurophysiology and may help affirm or disprove a compression neuropathy $[10,11]$. Study of changes in median nerve F-wave parameters after treatment for idiopathic CTS might be a supportive argument for the effectiveness of steroid treatment.

Furthermore, some studies have considered the sympathetic skin response (SSR) findings in CTS [12], but have not included follow-up of local steroid injection into the carpal tunnel. Recording of SSR in CTS may help to assess the viability of sympathetic sudomotor fibers and evaluate the response to steroid treatment.

In this study, effectiveness of local corticosteroid injection was measured nerve conduction studies (NCSs), median nerve F-wave parameters, and SSR on initial visit and at second month after treatment.

\section{Materials and methods}

NCSs were performed according to the American Association of Electrodiagnostic Medicine guidelines [6, 13] with a Medelec Teca Premerie Plus vE05 electromyograph (Surrey, UK) in all cases by the same person. All the tests were done in similar temperature conditions, and when the hands were cold, they were warmed. Ten consecutive patients (17 hands) with electrodiagnostically confirmed CTS and 21 control subjects with bilateral normal NCSs were prospectively evaluated. In the analysis all affected hands were included. Only mildly or moderately affected individuals were included in the study; "mild" describes patients with decreased mSNCV over the second digit-wrist segment and prolonged median sensory distal latency (mSDL), with sensory nerve action potentials amplitude (SNAPa) below the lower limit of normal; "moderate" describes patients with abnormally delayed mMDL and mSDL with decreased SNAPa [14].

We considered the median sensory distal latency (mSDL; normal value $\leq 3.5 \mathrm{~ms}$ ) and the $\mathrm{mSNCV}$ (normal value $\geq 46.5 \mathrm{~m} / \mathrm{s}$ ) or the $\mathrm{mMDL}$ (normal value $\leq 3.8 \mathrm{~ms}$ ) as the main tests for CTS diagnosis. A secondary criterion was the comparison with the median versus ulnar wrist-to-digit
4 sensory latencies (D4M-D4U). In this study, a D4M-D4U above to $0.45 \mathrm{~ms}$ was considered abnormal. The abnormal cutoff values for NCSs parameters were calculated as plus or minus 2 standard deviations from the mean values of control group.

Ten consecutive $\mathrm{F}$ waves were recorded for each median nerve. For each extremity, we calculated minimum (Fmin), maximum (Fmax), and mean (Fmean) F-wave latencies, frequency of the F-wave (Fp; F-persistence), chronodispersion $\left(\mathrm{F}_{\mathrm{CD}}\right)$, mean $\mathrm{F} / \mathrm{M}$ amplitude ratios $(\mathrm{mF} / \mathrm{M}$-amp), and F-wave conduction velocity (FwCV). The amplitude of each $\mathrm{F}$ response was measured from peak-to-peak. Among all onset latencies recorded, Fmin was the shortest and Fmax the longest. Fp was defined as the number of such Fresponses obtained with 10 stimuli, Fmean was defined as the mean onset latency of such F-responses, $\mathrm{F}_{\mathrm{CD}}$ was defined as the difference between Fmax and Fmin. Based on Fmin latency, FwCV was calculated using the previously described formula: $\mathrm{D} \times 2 /($ Fmin-mMDL-1), where $\mathrm{D}$ is the estimated distance from the stimulus site to the spinal cord [15].

Fourteen consecutive SSR were recorded for each median nerve. The unilateral median nerve was stimulated at the wrist with square electrical pulses of $0.2 \mathrm{~ms}$ duration and $20 \mathrm{~mA}$ intensity. SSR latencies, amplitudes, areas, and waveforms were determined for the patient and control groups. SSR waveforms were analyzed according to Toyokura [16] classification: responses in which one of the phases accounted for at least $80 \%$ of the peak-to-peak amplitude could be either positive (P) or negative (N) types.

The local steroid injection was given using a 23-gauge needle at proximal of the carpal tunnel to the wrist crease just medial to the tendons of the flexor radial muscle involving a single $1-\mathrm{ml}$ dexamethasone sodium phosphate (Dekort ${ }^{\circledR}$, Deva) injection without local anesthetic in affected hands. The needle was introduced slowly, and the patient was instructed to say stop if she felt needles or pain in the fingers.

\section{Statistical analysis}

For statistic analysis, we used the SPSS package 10.0.7 for Windows XP. The means and standard deviations (SD) were calculated for all subjects in each group for each parameter. Wilcoxon signed ranks test was used to compare before and after treatment values in patients with CTS. Mann-Whitney $U$ test was used to compare the differences among the values between CTS and control groups. We determined the percentage of patients that statistical improved by more than one standard deviation of the conduction values after the treatment. A probability $(P)$ value of less than 0.05 was considered significant. 


\section{Results}

The mean age was $46 \pm 6.4$ years in CTS group and $44.2 \pm 8$ years in controls; no significant difference was present in terms of age $(P>0.05)$. CTS was bilateral in 7 ( 14 hands, $82.4 \%$ ), on right hand in 2 cases and on the left side in 2 cases. Table 1 shows demographic characteristic of patient and control groups. According to the NCSs, CTS was mild in $8(47 \%)$ and moderate in $9(53 \%)$ hands of the patients.

The conventional motor and sensory NCSs of the median nerve were statistically different between the CTS patients and the controls (Table 2). At follow-up, NCSs of the median nerve showed a significant improvement with respect to baseline values. According to the reference value $\pm 1 \mathrm{SD}$, the recovery rates $(\%)$ of the D4M-D4U, mSDL, and mMDL were 65,35 , and $30 \%$ at 2-month follow-up, respectively.

Table 1 Demographic characteristic of patient and control groups

\begin{tabular}{llll}
\hline & CTS group & Controls & $P$ \\
\hline Sex (female) & $9(100 \%)$ & $21(100 \%)$ & NS \\
Age (mean \pm SD) & $46 \pm 6.4$ & $44.2 \pm 8$ & 0.337 \\
Height $($ mean \pm SD) & $161 \pm 12.3$ & $163 \pm 11.4$ & 0.855 \\
Weight (mean \pm SD) & $69.5 \pm 11.6$ & $66.7 \pm 8.0$ & 0.352 \\
Body mass index & $27.6 \pm 4.2$ & $26.7 \pm 3.2$ & 0.378 \\
\hline
\end{tabular}

The CTS patients showed prolonged F-wave latencies, decreased $\mathrm{Fp}$, and an increase in the $\mathrm{F}_{\mathrm{CD}}$ when compared with controls. As shown in Table 2, comparing other F-wave parameters between the CTS and the control groups did not reveal any statistically significant difference. At the end of the study, there was an improvement on Fmin, Fmax, and Fmean latencies, Fp, mF/M-amp, maximum, and mean F-wave amplitudes, but statistical significance was not achieved $(P>0.05)$. Also, the chronodispersion showed a slightly improvement at 2 months after treatment with respect to baseline values $(P=0.05)$ (Fig. 1).

SSR was obtained in all the controls and CTS patients, with a mean occurrence rate of 95.1 versus $90.3 \%$, respectively, $(P<0.05)$. In CTS patients, there were many absent responses $(9.7 \pm 14.7 \%)$; whereas in the control group, there was a little habituation percent of SSR $(4.9 \pm 10.5 \%)$. There were also less monophasic $\mathrm{P}$ responses (Fig. 2) in the CTS group (41.2\% of responses versus. $47.9 \%$ in the control group). These differences, however, did not reach statistical significance. Also, as shown in Table 3, comparing other SSR parameters between the CTS and the control groups did not reveal any statistically significant difference at baseline. No significant differences in SSR wave types, latencies and areas were revealed at 2 months after treatment with respect to baseline values $(P=0.05)$. Although no statistically significant differences were observed in SSR parameters, slightly decreased amplitudes and increased habituation of SSR were noted at the end of the treatment.

Table 2 The mean values and standard deviations of the NCSs and F-wave parameters in CTS before and after the treatment and controls groups

\begin{tabular}{|c|c|c|c|c|c|}
\hline Parameters & $\begin{array}{l}\text { Controls } \\
(n=42 \text { hands })\end{array}$ & $\begin{array}{l}\text { Patients } \\
\text { (before) } \\
\text { ( } n=17 \text { hands) }\end{array}$ & $\begin{array}{l}\text { Patients } \\
\text { (after) } \\
(n=17 \text { hands) }\end{array}$ & $\begin{array}{l}P 1 \\
\text { (controls a } \\
\text { nd patients) }\end{array}$ & $\begin{array}{l}P 2 \text { (before } \\
\text { and after } \\
\text { treatment) }\end{array}$ \\
\hline \multicolumn{6}{|l|}{ Nerve conduction studies } \\
\hline Distal motor latency of median nerve (ms) & $3.1 \pm 0.4$ & $4.2 \pm 0.7$ & $4.0 \pm 0.4$ & $<0.001$ & $<0.05$ \\
\hline The median sensory nerve latency (ms) & $3.1 \pm 0.2$ & $4.1 \pm 0.4$ & $3.8 \pm 0.3$ & $<0.001$ & $<0.01$ \\
\hline $\mathrm{mSNCV}$ on the second digit & $46.5 \pm 2.9$ & $34.6 \pm 3.3$ & $36.3 \pm 2.4$ & $<0.001$ & $<0.01$ \\
\hline D4M-D4U (ms) & $0.16 \pm 0.14$ & $1.66 \pm 0.65$ & $1.31 \pm 0.41$ & $<0.001$ & $<0.001$ \\
\hline \multicolumn{6}{|l|}{ F waves parameters } \\
\hline Minimum F-wave latency (ms) & $23.6 \pm 1.2$ & $26.0 \pm 2.6$ & $26.1 \pm 2.6$ & $<0.001$ & $N S$ \\
\hline Maximum F-wave latency (ms) & $26.1 \pm 1.6$ & $29.4 \pm 2.5$ & $29.1 \pm 2.7$ & $<0.001$ & $N S$ \\
\hline Mean latency of F-wave (ms) & $24.7 \pm 1.4$ & $27.3 \pm 2.6$ & $27.4 \pm 2.5$ & $<0.001$ & $N S$ \\
\hline F-wave chronodispersion (ms) & $2.5 \pm 1.1$ & $3.4 \pm 0.96$ & $3.0 \pm 0.65$ & $<0.01$ & 0.05 \\
\hline F-wave persistence $(\%)$ & $93.8 \pm 7.3$ & $85.9 \pm 12.3$ & $87.7 \pm 11.5$ & $<0.01$ & $N S$ \\
\hline Mean F/M amplitude ratios & $0.035 \pm 0.02$ & $0.049 \pm 0.06$ & $0.042 \pm 0.03$ & $N S$ & $N S$ \\
\hline F-wave conduction velocity $(\mathrm{m} / \mathrm{s})$ & $73.1 \pm 8.0$ & $70.3 \pm 6.8$ & $69.6 \pm 9.8$ & $N S$ & $N S$ \\
\hline Maximum F-wave amplitude $(\mu \mathrm{V})$ & $0.490 \pm 0.21$ & $0.510 \pm 0.21$ & $0.553 \pm 0.30$ & $N S$ & $N S$ \\
\hline Mean F-wave amplitude $(\mu \mathrm{V})$ & $0.279 \pm 0.11$ & $0.290 \pm 0.10$ & $0.310 \pm 0.15$ & $N S$ & $N S$ \\
\hline
\end{tabular}

$m S N C V$ median sensory nerve conduction velocity, $N S$ not significant, $D 4 M-D 4 U$, the median versus ulnar wrist-to-digit 4 sensory latencies 

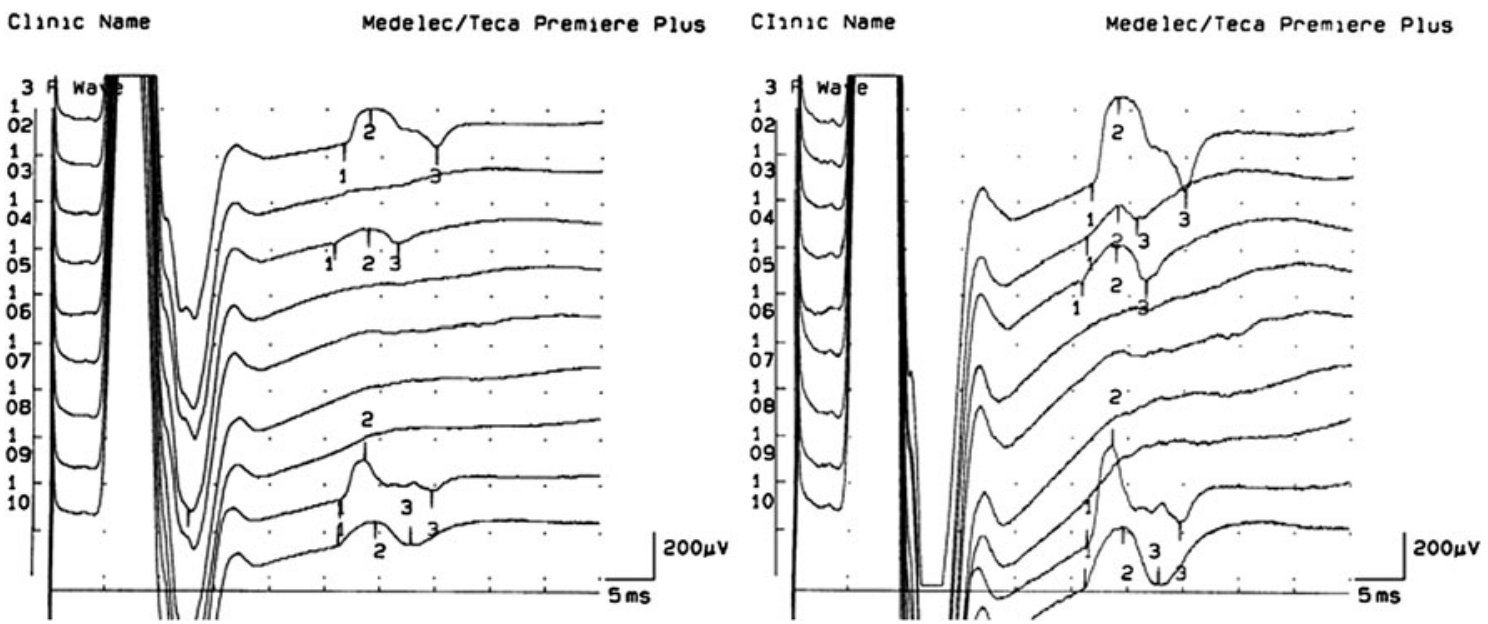

Fig. 1 Example of $\mathrm{F}$ waves before and after treatment
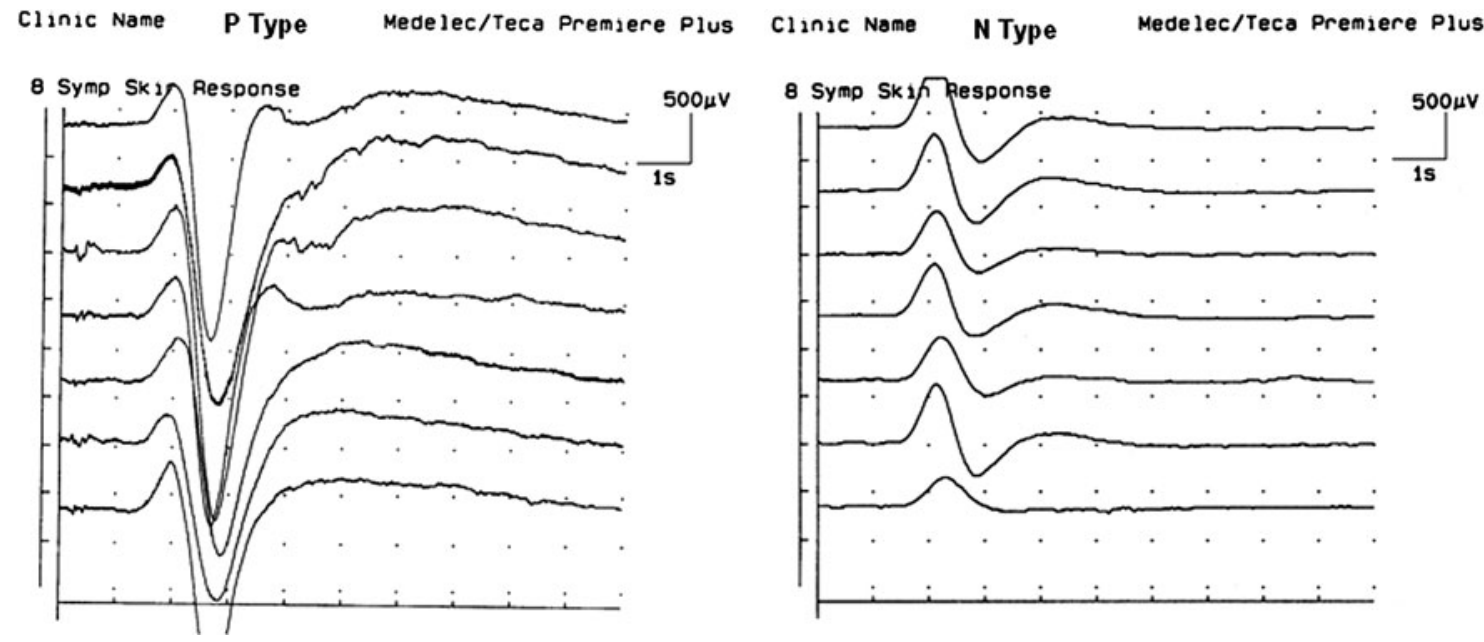

Fig. 2 Sympathetic skin response wave types

\section{Discussion}

To elucidate better the effectiveness of conservative treatment, several controlled prospective studies using clinical and electrophysiological data have been performed. Steroid injections are known to be a safe and effective treatment for relief of symptoms associated with CTS in patients. It can lead to significant improvement of both NCSs and clinical parameters [5, 7, 17-19]. Steroids are effective at reducing swelling on account of their anti-inflammatory action [4]. Several tools have been used to assess the outcome of CTS treatment. Of most interest are the NCSs findings. We used electrodiagnostic measurements for an objective assessment. Steroid injection provides an improvement of particularly sensory NCS [5, 7]. This study shows that the latency differences after sensory median-ulnar techniques are better than the routine median sensory and motor latencies at follow-up. Though reported to be effective, there are controversies on the role of local steroid injection with recurrence rates given from 8 to $100 \%$. Girlanda et al. [19] assessing local steroid treatment, the follow-up by means of clinical and electrophysiological examinations, reported that $50 \%$ of the nerves became worse within 6 months. This condition could be attributed to a decrease in the effectiveness of the theraphy and the progression of disease by time. The results of our study similarly to previous studies $[5,7$, 17-19] suggest that with mild to moderate CTS cases are good candidates for this type of treatment, and the treatment of CTS with low-dose dexamethasone sodium phosphate injection is effective in short-term follow-up.

In the present study, the nerve conduction velocity improved significantly at the end of the study. However, comparing F-wave parameters did not reveal any statistically significant difference before and after treatment in this study. The maximum and mean $\mathrm{F}$-wave amplitudes and $\mathrm{F}_{\mathrm{CD}}$ showed a slight improvement with respect to baseline values 
Table 3 The mean values and standard deviations SSR parameters in CTS before and after the treatment and controls groups

\begin{tabular}{|c|c|c|c|c|c|}
\hline Parameters & $\begin{array}{l}\text { Controls } \\
(n=42 \text { hands })\end{array}$ & $\begin{array}{l}\text { Patients } \\
\text { (before) } \\
\text { ( } n=17 \text { hands) }\end{array}$ & $\begin{array}{l}\text { Patients } \\
\text { (after) } \\
(n=17)\end{array}$ & $\begin{array}{l}P 1 \\
\text { (controls } \\
\text { and patients) }\end{array}$ & $\begin{array}{l}P 2 \text { (before } \\
\text { and after } \\
\text { treatment) }\end{array}$ \\
\hline \multicolumn{6}{|c|}{ Sympathetic skin response } \\
\hline $\mathrm{N}$ type waves $(\%)$ & $47.4 \pm 33.0$ & $48.7 \pm 35.1$ & $48.1 \pm 31.2$ & $N S$ & 0.48 \\
\hline P type waves (\%) & $47.9 \pm 34.1$ & $41.2 \pm 38.2$ & $37.1 \pm 37.8$ & $N S$ & 0.17 \\
\hline Mean amplitude & $0.816 \pm 0.46$ & $0.762 \pm 0.42$ & $0.608 \pm 0.39$ & $N S$ & 0.17 \\
\hline Maximum amplitude & $1.402 \pm 0.78$ & $1.325 \pm 0.72$ & $0.995 \pm 0.62$ & $N S$ & 0.26 \\
\hline Amplitude ratio & $0.69 \pm 0.48$ & $0.60 \pm 0.46$ & $0.67 \pm 0.35$ & $N S$ & 0.64 \\
\hline Habituation (\%) & $4.9 \pm 10.5$ & $9.7 \pm 14.7$ & $12.2 \pm 20.5$ & $N S$ & 0.68 \\
\hline Mean latency & $1.46 \pm 0.13$ & $1.42 \pm 0.12$ & $1.45 \pm 0.13$ & $N S$ & 0.21 \\
\hline Maximum latency & $1.61 \pm 0.16$ & $1.59 \pm 0.17$ & $1.65 \pm 0.16$ & $N S$ & 0.09 \\
\hline Mean area & $1.260 \pm 0.58$ & $1.135 \pm 0.54$ & $1.130 \pm 0.80$ & $N S$ & 0.96 \\
\hline Maximum area & $2.264 \pm 1.10$ & $2.071 \pm 1.05$ & $1.689 \pm 1.09$ & $N S$ & 0.31 \\
\hline
\end{tabular}

$m S N C V$ median sensory nerve conduction velocity, $N S$ not significant, $D 4 M-D 4 U$, the median vs. ulnar wrist-to-digit 4 sensory latencies

and controls, but statistical significance was not achieved. This change may be due to reinnervation of median nerve. Panayiotopoulos et al. [10] point out that the amplitude of $\mathrm{F}$ waves is much higher in reinnervation of peripheral neuropathies and low in muscular dystrophies. Todnem et al. studied recovery of median nerve function in operated elderly and younger patients with carpal tunnel syndrome, using neurophysiological methods. They found that F-responses were significantly reduced in the hands of younger operated adults, but not in the elderly subjects undergoing operation, probably due to the smaller number of operated wrists in the latter group [20]. Non-operated hands also had significantly shorter F-responses at the second examination in their study [20], probably caused by unblocking of the fastest nerve fibers in the carpal tunnel [21]. We suggest that NCSs may improve in many patients in whom F-wave parameters do not return to normal. Thus, the NCSs are better than that shown by F-wave parameters. The F-wave parameters were not effectual to assess the median nerve recovery after treatment.

SSR itself has no value for detecting CTS [13] but its value for assessing autonomic disturbances in CTS is conflicting [22-25]. In our study, SSR was obtained in all the controls and CTS patients, with a mean occurrence rate of 95.1 versus $90.3 \%$, respectively. There were many absent responses in CTS patients. We also investigated the wave types and observed lesser P-type SSR in CTS hands than controls. These differences, however, did not reach statistical significance. SSR waveforms have been studied in normal subjects and CTS patients in recent years [16, 23, 25, 26]. Brazil-Neto [25] found significant changes in wave types in idiopathic CTS. According to a recent theory, P-type reflects stronger activation of a large number of sweat glands [27]. $\mathrm{N}$ responses, according to Toyokura [16], tend to appear late in a series of SSR, which suggests they may be produced by habituation. In this study, although no statistically significant improvement was observed in SSR parameters, slightly decreased amplitudes and increased habituation of SSR were noted at the end of the treatment. Previous studies [26] showed a marked decrease in the SSR amplitude or occurrence rate in some subjects on the second examination. These findings suggest that some subjects experienced earlier development of habituation in the second test in CTS [26]. The absence of improvement in SSR after treatment may be due to the poor reinnervation capacity of sympathetic fibers after chronic compression [12]. On the other hand, SSR does not seem to be a sensitive method for evidence of autonomic involvement in CTS, even in patients manifesting sudomotor or other autonomic symptoms [28].

In conclusion, the present study showed that the local steroid injection resulted improvement in NCSs values, but not in SSR and F-wave parameters. The SSR and F waves do not seem to be a sensitive method for predicting outcome in short-term follow-up in CTS treatment. Mild to moderate CTS cases are good candidates for this type of treatment, and the treatment of CTS with low-dose dexamethasone sodium phosphate injection is effective in short-term follow-up.

Open Access This article is distributed under the terms of the Creative Commons Attribution Noncommercial License which permits any noncommercial use, distribution, and reproduction in any medium, provided the original author(s) and source are credited.

\section{References}

1. Atroshi I, Gummesson C, Johnsson R, Ornstein E, Ranstam J, Rosén I (1999) Prevalence of carpal tunnel syndrome in a general population. JAMA 282:153-158 
2. Ferry S, Pritchard T, Kenan J, Croft P, Silman AJ (1998) Estimating the prevalence of delayed median nerve conduction in the general population. Br J Rheumatol 37:630-635

3. Gerritsen AA, de Krom MC, Struijs MA, Scholten RJ, de Vet HC, Bouter LM (2002) Conservative treatment options for carpal tunnel syndrome: a systematic review of randomised controlled trials. J Neurol 249(3):272-280

4. Aroori S, Spence RAJ (2008) Carpal tunnel syndrome. Ulster Med J 77(1):6-17

5. Ayhan-Ardic F, Erdem H (2000) Long-term clinical and electrophysiological results of local steroid injection in patients with carpal tunnel syndrome. Funct Neurol 15:157-165

6. Jablecki CK, Andary MT, Floeter MK, Miller RG, Quartly CA, Vennix MJ, Wilson JR (2002) Second AAEM literature review of the usefulness of nerve conduction studies and needle electromyography for the evaluation of patients with carpal tunnel syndrome. Muscle Nerve 26(suppl):S1-S53

7. Hagebeuk EEO, de Weerd AW (2004) Clinical and electrophysiological follow-up after local steroid injection in the carpal tunnel syndrome. Clin Neurophysiol 115(6):1464-1468

8. Uncini A, Di Muzio A, Awad J, Manante G, Tafuro M, Gambi D (1993) Sensitivity of three median to ulnar comparative tests in diagnosis of mild carpal tunnel syndrome. Muscle Nerve 16:1366-1373

9. Chang MH, Liu LH, Lee YC, Wei SJ, Chiang HL, Hsieh PF (2006) Comparison of sensitivity of transcarpal median motor conduction velocity and conventional conduction techniques in electro diagnosis of carpal tunnel syndrome. Clin Neurophysiol 117(5):984-991

10. Panayiotopoulos CP, Chroni E (1996) F-waves in clinical neurophysiology: a review, methodological issues and overall value in peripheral neuropathies. Electromyogr Clin Neurophysiol 101:365-374

11. Fisher MA, Hoffen B (1997) F wave analysis in patients with carpal tunnel syndrome. Electromyogr Clin Neurophysiol 37(1):27-31

12. Mondelli M, Vecchiarelli B, Reale F, Marsili T, Giannini F (2001) Sympathetic skin response before and after surgical release of carpal tunnel syndrome. Muscle Nerve 24:130-133

13. American Association of Electrodiagnostic Medicine, American Academy of Neurology, American Academy of Physical Medicine and Rehabilitation (2002) Practice parameter for electrodiagnostic studies in carpal tunnel syndrome: summary statement. Muscle Nerve 25:918-922
14. Stevens JC (1997) AAEM minimonograph \#26: the electrodiagnosis of carpal tunnel syndrome. Muscle Nerve 20:1477-1486

15. Kimura J (1989) Electrodiagnosis in diseases of nerves and muscles: principles and practice. F.A. Davis, Philadelphia, pp 332-353

16. Toyokura M (1998) Waveform and habituation of sympathetic skin response. Clin Neurophysiol 109(2):178-183

17. Armstrong T, Devor W, Borschel L, Contreras R (2004) Intracarpal steroid injection is safe and effective for short-term management of carpal tunnel syndrome. Muscle Nerve 29(1):82-88

18. Demirci S, Kutluhan S, Koyuncuoglu HR, Kerman M, Heybeli N, Akkus S, Akhan G (2002) Comparison of open carpal tunnel release and local steroid treatment outcomes in idiopathic carpal tunnel syndrome. Rheumatol Int 22(1):33-37

19. Girlanda P, Dattola R, Venuto C, Mangiapane R, Nicolosi C, Messina C (1993) Local steroid treatment in idiopathic carpal tunnel syndrome: short- and long-term efficacy. J Neurol 240(3):187-190

20. Todnem K, Lundemo G (2000) Median nerve recovery in carpal tunnel syndrome. Muscle Nerve 23:1555-1560

21. Wilson RW (1998) Median mixed nerve conduction studies in the forearm: evidence against retrograde demyelination in the carpal tunnel. J Clin Neurophysiol 15:541-546

22. Verghese J, Galanopoulou AS, Herskovitz S (2000) Autonomic dysfunction in idiopathic carpal tunnel syndrome. Muscle Nerve 23:1209-1213

23. Kiylioglu N, Akyol A, Guney E, Bicerol B, Ozkul A, Erturk A (2005) Sympathetic skin response in idiopathic and diabetic carpal tunnel syndrome. Clin Neurol Neurosurg 108(1):1-7

24. Kanzato N, Komine Y, Kanaya F, Fukiyama K (2000) Preserved sympathetic skin response at the distal phalanx in patients with carpal tunnel syndrome. Clin Neurophysiol 111(11):2057-2063

25. Brasil-Neto JP, Carneiro CR (2004) Monophasic negative sympathetic skin responses and autonomic dysfunction in carpal tunnel syndrome. Muscle Nerve 29(2):330-331

26. Toyokura M, Murakami K (1996) Reproducibility of sympathetic skin response. Muscle Nerve 19(11):1481-1483

27. Mitani H, Ishiyama Y, Hashimato I (2003) Equivalent current dipole estimated from SSR potential distribution over the human hand. Clin Neurophysiol 114:233-238

28. Argyriou AA, Polychronopoulos P, Moutopulou E, Aplada M, Chroni E (2006) The significance of intact sympathetic skin responses in carpal tunnel syndrome. Eur J Neurol 13:455-459 